\title{
Laser diffractometer for single-particle scattering measurements
}

\author{
Z Ulanowski,RSGreenaway, P H Kaye and I K Ludlow \\ Department of Physical Sciences, University of Hertfordshire, Hatfield, Hertfordshire AL10 9AB, \\ UK \\ E-mail: z.ulanowski@herts.ac.uk
}

Received 2 August 2001, in final form 6 December 2001, accepted for publication 4 January 2002 Published 8 February 2002

\begin{abstract}
A laser diffractometer (polar nephelometer) constructed for single-particle studies is described. It takes advantage of an array of optical fibres and a scanning disc to allow the use of a single photomultiplier tube for the detection of scattered light. A background subtraction scheme makes the instrument especially valuable in cases where background scattering exceeds the scattering from the observed particle, for example for living cells in aqueous media. The system can be easily integrated with particle trapping equipment such as laser tweezers or an electrodynamic balance.
\end{abstract}

Keywords: light scattering, single particle, laser diffractometry, optical trapping levitation, refractive index, cell, spore, water content

\section{Introduction}

Laser diffractometery, sometimes known as polar nephelometry or multiangle photometry, is concerned with the measurement of angular distribution of light scattered from an object, whether a single small particle, an ensemble of particles or a macroscopic specimen (Chu 1991). The technique can give information about the size, refractive index and coarse internal structure of small particles, such as living cells. In the case of cells, the refractive index can be converted into solid or water content using simple relationships. If an assumption is made that the structure of the cells can be approximated by a inhomogeneous model such as the coated sphere, the distribution of water can be measured in a non-invasive way (Ulanowski et al 1987, Ulanowski and Ludlow 1989). A field of fundamental as well as practical importance is scattering from non-spherical particles; within this area scattering from atmospheric ice crystals has recently grown in significance because of the impact it has on climate (see e.g. Bacon and Swanson 2000, Barkey and Liou 2001, Ulanowski et al 2002). A valuable addition to laser diffractometry is particle trapping, as it allows prolonged measurements of particles in isolation from any solid supports, as well as measurements on aligned particles. In liquid media single-beam optical gradient traps (laser tweezers) can be applied (Ulanowski and Ludlow 2000, Doornbos et al 1996) while for airborne particles electrodynamic trapping is useful (Hesse et al 2002a, Bacon and Swanson 2000).

In the following sections the detailed design of a diffractometer designed specifically for single-particle studies is described.

\section{System description}

The diffractometer described here is an improved version of previously published designs (Kaye and Ludlow 1979, Ludlow and Kaye 1979, Ulanowski 1988). It uses a wide-angle, semi-circular fibre-optics array for fast collection of multi-angle scattering data, coupled to a scanning system allowing the use of a single photomultiplier tube (PMT) as a detector (figure 1). A multi-wavelength laser functions as a light source. The data acquisition computer also controls functions such as the wavelength and state of polarization of incident light. Consequently, at least in the case of immobilized (trapped) particles, it is possible to carry out several measurements on a single particle, e.g. using different wavelengths and/or states of polarization. 
Provision has been made for subtracting background scattering (i.e. scattering in the absence of the object of interest) from the measured signal

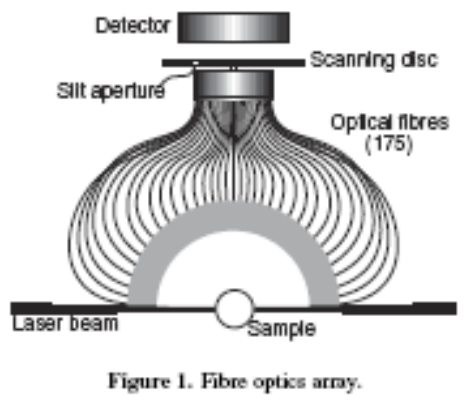

\subsection{Optical system}

A key element of the instrument is a semi-circular array of 175 optical fibres arranged radially in the horizontal plane around the scattering volume (figure 1). Each fibre subtends an angle of $1^{\circ}$ and the whole array extends over $3^{\circ}-177^{\circ}$ relative to the direction of the incident beam provided by a laser. The fibres at the input end are held in one plane by sandwiching them between two flat, arc-shaped plastic 'jaws'. One of these has radial grooves for retaining the fibres and was cast in epoxy resin using a precision-machined metal mould. The fibres are polymer ones with a diameter of $0.5 \mathrm{~mm}$; both fibre ends are coated with acrylic varnish to improve their uniformity. Thus the light scattered by a particle within the laser beam and in the field of view of the array will enter the fibres and subsequently emerge from the output ends. These ends are arranged parallel to each other in a cylindrical configuration (figure 2). The output faces of the fibres are scanned using a rotating, opaque disc with a slit allowing light from only one fibre to pass through at any time, on average. The scattered light can thus be conveyed sequentially to a single optical detector-in this case a photomultiplier tube (PMT, Philips XP2203B, chosen for its large photocathode and red light sensitivity). In this way the angular light-intensity pattern is converted into a time-dependent electrical signal, which can be further processed. The scanning disc is also provided with a peripheral array of alternate opaque and transparent bars, with an angular spacing identical to the spacing of the output ends of the fibres ( $\left.2^{\circ}\right)$, and a single reflective stripe (figure 3 ). While the disc is rotating the bars and the stripe, together with appropriate optical sensors, produce timing pulses necessary for relating the time-dependent signal to the corresponding scattering angle. A hollow cylindrical reflector is inserted between the fibres and the PMT so as to maximize optical throughput. An optical filter can be placed between the mirror and the PMT if it is desirable to remove unwanted wavelengths, for example from specimen fluorescence or a trapping laser beam. The duration of a complete angular scan is controlled by setting the speed of a de motor (ironless rotor, Maxon, Switzerland) to which the scanning disc is attached. The lower scanning time limit is at present $10 \mathrm{~ms}$, allowing up to 100 patterns to be collected per second.

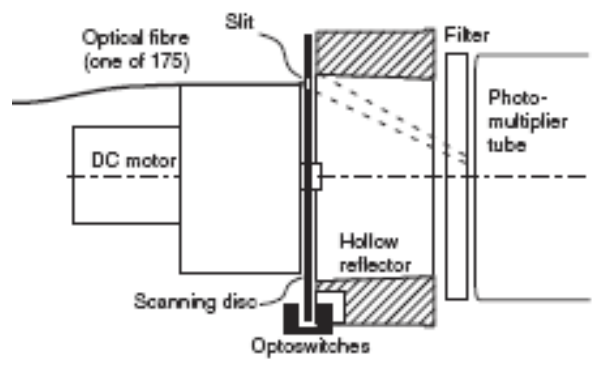

Figure 2. Fibre amay scanning amangement.

The incident beam is provided by a multi-line argon-ion laser. Wavelength selection is accomplished using a prism placed on a rotation stage, the angle of which is controlled by a stepper motor driven from the data acquisition computer. The state of polarization of the beam can be altered using a multiline, quartz retarder plate. With a quarter-wave plate linear and circular beam polarization can be produced; with a half-wave plate two (or more) orthogonal linear polarizations can be obtained. The instantaneous power of the beam is monitored

using a beam-splitter, which directs part of the beam into an optical fibre. The output from the fibre is then detected using a photodiode and amplified, so that it can be used to compensate for variations in the beam power (see section 2.3 for further details). The beam can be spatially filtered, expanded and focused to an appropriate diameter, as required by the size and nature of the specimen (beams with diameters between 30 and $800 \mu \mathrm{m}$ have been used). The beam transmitted past the specimen is dumped in a light-trap. 


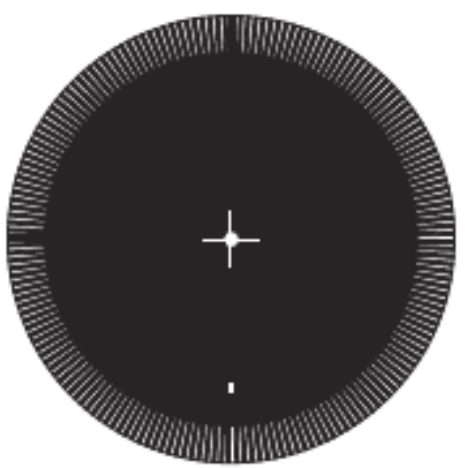

Figure 3. Scanning disc, showing the scanning slit and 190 timing

bars (see text).

\subsection{Data acquisition}

A schematic diagram of the diffractometer is shown in figure 4. The main data acquisition function is, of course, input data sampling and storage. This is carried out concurrently with outputting stored scattering background subtraction terms and correction factors adjusting for non-uniformity of the fibre optics array. Following the conversion of the scattered intensity into a time-dependent current, as described in the preceding section, the microcomputer samples the incoming signal using a 12-bit analogue to digital converter, the timing being determined by the strobe pulses from the scanning disc. The signal can be conditioned using an active, computer-controlled low-pass filter. Transitions between measurement points are masked using a sample and hold circuit. There is a choice of linearly or logarithmically scaled signal, the latter being provided by a high-speed logarithmic amplifier, giving better signal resolution at lower signal levels.

The use of a large number of optical fibres requires some means of correcting the signal for attenuation caused by differences in the properties of the fibres (transmittance and end-face finish). Unless such compensation is carried out, a pseudo-random envelope will be imposed on the output signal, preventing correct interpretation of the light-scattering pattern. The signal correction is achieved by the use in the signal path of an 12-bit digital to analogue (D/A) converter functioning in a multiplication mode. Predetermined correction factors, stored in the microcomputer, are used to drive the converter synchronously with the arrival of the signal corresponding to the output from individual optical fibres, thus enabling the attenuation of the signal by the correct amount for each fibre. The correction factors are initially measured by inserting a rotating, linear-filament, incandescent light bulb in the centre of the array in place of the specimen and measuring the output signal over several hours. A similar measurement could probably be carried out using a rotating diffuser coupled to an optical fibre (Barkey and Liou 2001). The inverse of the averaged output for each angular data point then becomes the correction factor for the corresponding fibre.

The scattering patterns are displayed (in the angle- intensity coordinates) by outputting an analogue signal to a digitizing oscilloscope during both data acquisition and data analysis. In the data acquisition mode this form of display makes possible real-time observations of the incoming data, thus avoiding possible software and hardware conflicts between real-time data acquisition and graphical data display on a computer monitor.

\subsection{Background subtraction}

In the case of measurements carried out on single particles, especially in liquid media, the scattering intensity from the suspending medium can be comparable in magnitude to, or much higher than, that from the particle, even in the absence of any impurities (Ulanowski 1988). Providing some means of removing (subtracting) the background component from the light-scattering patterns is therefore highly advantageous. In the present system the subtraction is carried out at the earliest possible stage of signal processing, namely at the PMT output, by subtracting the current produced by a 16-bit D/A converter from the anode current. The converter is driven using a set of subtraction terms, one for each discrete scattering angle, generated during background measurements (in the absence of the measured particle) and stored in the microcomputer.

Since the background scattering may be so high as to saturate the electronic circuits, an iterative procedure is applied to generating the subtraction terms. The background is measured in a sequence of discrete steps and each time the measured background is added to the one measured in the previous step. To avoid over-subtraction of the background, which would result in negative scattered intensity values, at each step a set of several measurements is collected for each scattering angle and the lowest value only is used for calculating the background subtraction terms.

The subtraction circuit is also used for the compensation of the noise generated by variations of the output power of the laser. When the background scattering is of a higher magnitude than the scattering from the observed particle, most noise tends 
to originate from fluctuations of the background, which, in turn, may have a major component due to laser noise. Removal of this component can be achieved by multiplying the background subtraction signal by a factor proportional to the intensity of the incident beam at any given moment. Since a multiplying D/A converter is already present in the background subtraction circuit it can used for this purpose (see figure 4). The signal derived from a photodiode monitoring the laser beam is amplified and fed into the reference input of the $\mathrm{D} / \mathrm{A}$ converter. The compensation circuit is also provided with means of normalizing the noise-compensation signal against the mean beam-power level.

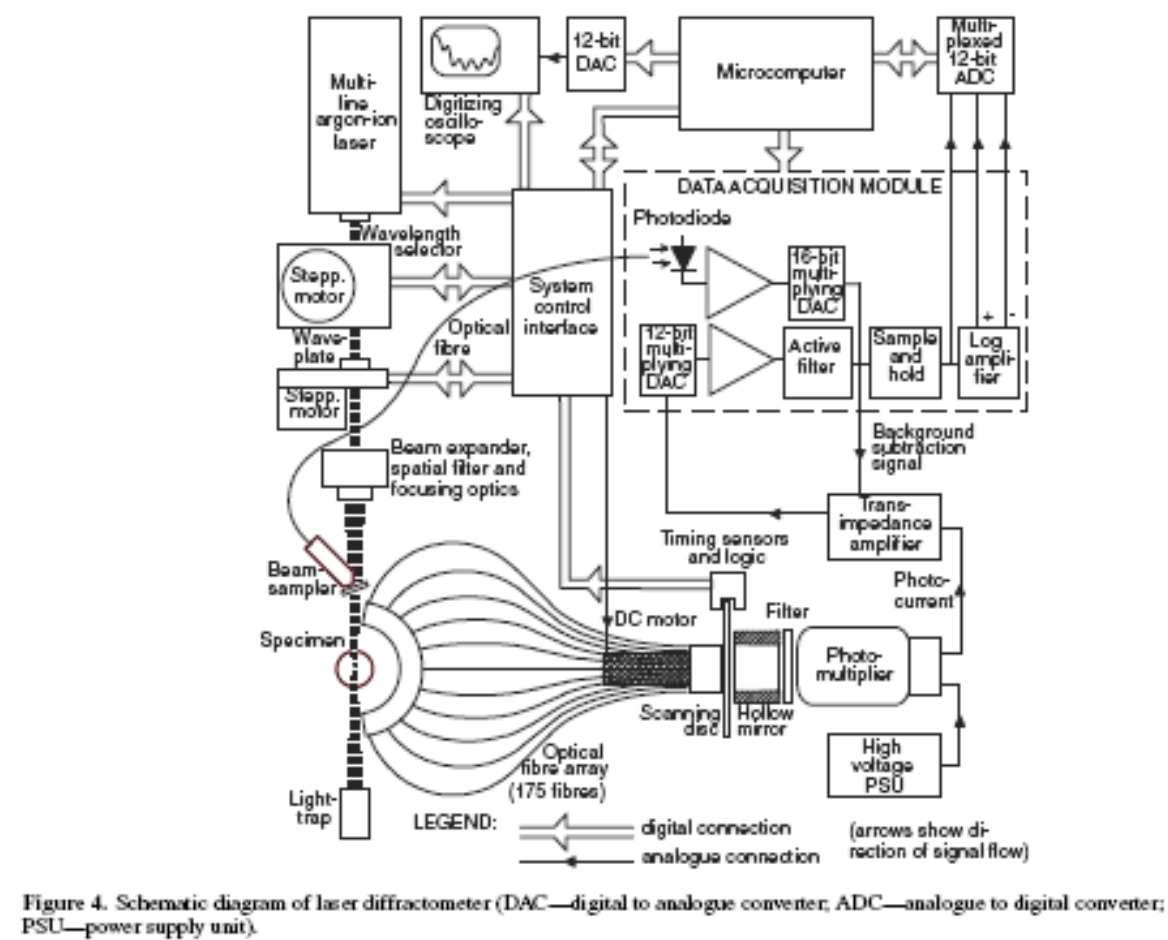

Figure 4. Schemaic diagr
PSU-power supply unit).

\section{Results and discussion}

The use of a fibre optics array in the diffractometer brings several advantages over 'classical' multi-angle photometer designs. Firstly, much faster scanning (up to 100 scattering patterns per second in the present system) is possible than in systems employing a moving detector. Secondly, good access to the scattering volume is available, making it possible to augment the system in various ways, for example by including a particle trap and/or observation microscopes. Thirdly, there is little possibility of misalignment, as timing and scanning functions are closely integrated. However, two disadvantages are also apparent: constructing the optical fibre array is relatively difficult and time consuming and the angular resolution of the system is inherently fixed and linear across the whole range of measurement angles.

The system is suitable for studies of small, single particles in both air and liquids. For example, studies using earlier embodiments of the system (based on essentially the same optical design) demonstrated the possibility of measuring the size and water content of protoplasts and, separately, integuments of individual, viable bacterial (Ulanowski et al 1987) and fungal (Ulanowski and Ludlow 1989) spores in an aqueous environment. In these studies cells with sizes down to less than one micrometre were presented using continuous flow systems (Ulanowski 1988). To allow work with immobilized particles, two separate trapping arrangements were combined with the diffractometer. For measurements on particles in water, for example living cells, a single-beam optical gradient trap (laser tweezers) was used (Ulanowski and Ludlow 2000), while for airborne particles an electrodynamic balance was constructed (Hesse et al 2002a). Such non-contact particle trapping is a valuable enhancement, especially for studies of fundamental nature. On the one hand it can be used to fix the particle spatially and define its orientation, allowing long measurement times. On the other hand it also permits presentation of nonspherical particles at a variety of orientations (Hesse et al 2002a), the usefulness of the system being further enhanced in this context by the ability to provide data at different wavelengths and states of polarization, as recently demonstrated by measurements on trapped ice crystal analogues carried out using the instrument described here (Ulanowski et al 2002, Hesse et al 2002b). High-resolution imaging of trapped particles is also possible, either using optical microscopy during measurements, or following particle recovery from the trap, when electron microscopy and other forms of post-analysis can be applied (Hesse et al 2002a). An example scattering diagram (phase function) obtained using the system for a single polystyrene sphere with an approximate radius of $1.1 \mu \mathrm{m}$, immersed in water and illuminated with a laser beam polarized perpendicularly to the scattering plane with a wavelength of $0.5145 \mu \mathrm{m}$, is given in figure 5. The best-fit theoretical diagram shown in this figure was calculated from Lorenz-Mie theory, using radius and refractive index values obtained using a global stochastic optimization method (Zakovic et al 1998). 
The background subtraction scheme employed in the present system makes it especially valuable in cases where background scattering is stronger than the scattering from the observed particle, for example when objects such as living cells in an aqueous medium are observed. When the background signal is subtracted directly from the PMT anode current, the dynamic range of the measured intensity can be effectively extended by more than one order of magnitude. This is because the only part of the system having to accept the whole dynamic range of the signal is the PMT - a device characterized by excellent linearity, high sensitivity, low noise and high speed. Therefore, much less severe demands are put on the electronic signal amplification and digitization circuits. This brings a significant advantage over diffractometers based on multi-element detector arrays (see e.g. Chu et al 1992, Barkey and Liou 2001). Also, incorporating laser beam monitoring in the subtraction subsystem gives independence from laser noise. The benefit of the last feature is again most apparent in cases where strong background scattering is present, as high noise levels of some lasers (over $1 \%$ over relatively narrow bandwidths) could otherwise swamp useful signals.

In conclusion, an instrument useful for both applied and fundamental work in light scattering on single particles has been described. The benefits of the present system would appear to outweigh its disadvantages, including somewhat troublesome construction of the fibre optics array. The instrument is characterized by large dynamic range, the ability to 295 detect single-particle scattering in the presence of strong backgrounds, immunity from laser noise, ease of alignment and high data collection rate. It can be easily combined with optical or electrodynamic traps.

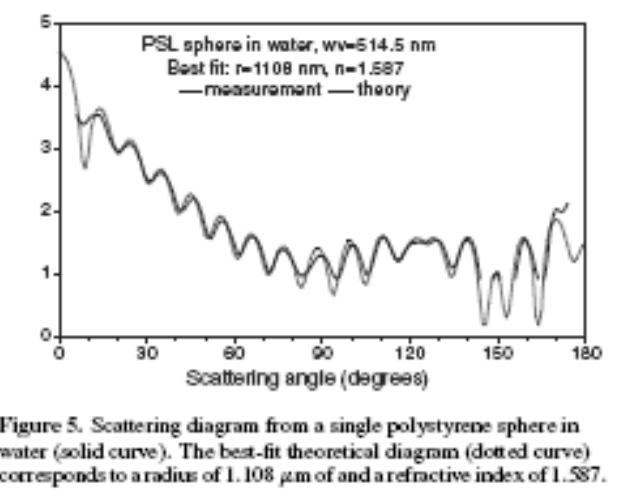

\section{Acknowledgments}

The authors are grateful to John Kerr for technical assistance and many practical ideas. This research was supported by the Engineering and Physical Sciences Research Council.

\section{References}

Bacon N J and Swanson B D 2000 J. Atmos. Sci. 57 2094-104 Barkey B and Liou K N 2001 Opt. Lett. 26 232-4 Chu B 1991 Laser Light

Scattering 2nd edn (New York: Academic) Chu B, Zhou Z and Moser H O 1992 Rev. Sci. Instrum. 63 2954-7

DoornbosRMP, Schaeffer M, Hoekstra A G, SlootPMA, de Grooth B G and Greve J 1996 Appl. Opt. 35 729-34 Hesse E, Ulanowski $\mathrm{Z}$ and Kaye P H 2002a J. Aerosol Sci. 33 149-63

Hesse E, Ulanowski Z and Baran A J 2002b 6th Int. Conf. on Electromagnetic and Light Scattering by Non-Spherical Particles (Gainesville)

Kaye P H and Ludlow I K 1979 J. Phys. E: Sci. Instrum. 12 690-3 Ludlow I K and Kaye P H 1979 J. Colloid Interface Sci. 69 571-89

Ulanowski Z 1988 Investigations of microbial physiology and cell structure using laser diffractometry $P h D$ Thesis CNAA

Ulanowski Z, Hesse E, Kaye P H, Baran A J, Chandrasekhar R and Parfitt C 2002 6th Int. Conf. on Electromagnetic and Light Scattering by Nonspherical Particles (Gainesville)

Ulanowski Z and Ludlow I K 1989 Mycol. Res. 93 28-32 Ulanowski Z and Ludlow I K 2000 Meas. Sci. Technol. 11 1778-85 Ulanowski Z, Ludlow I K and Waites W M 1987 FEMS Microbiol.

Lett. 40 229-32 Zakovic S, Ulanowski Z and Bartholomew-Biggs M C 1998 Inverse Problems 14 1053-67 